\title{
A single amino acid substitution (D1441Y) in the carboxyl-terminal propeptide of the proal (I) chain of type I collagen results in a lethal variant of osteogenesis imperfecta with features of dense bone diseases
}

\author{
J M Pace, D Chitayat, M Atkinson, W R Wilcox, U Schwarze, P H Byers
}

J Med Genet 2002;39:23-29

See end of article for authors' affiliations

.....................

Correspondence to: Dr P H Byers, Department of Pathology, University of Washington, Box 357470 Seattle, WA 98195, USA; pbyers@u.washington.edu

Revised version received 24 October 2001

Accepted for publication 26 October 2001

\begin{abstract}
Osteogenesis imperfecta $(\mathrm{OI})$ is characterised by brittle bones and caused by mutations in the type I collagen genes, COLIA 1 and COLIA2. We identified a mutation in the carboxyl-terminal propeptide coding region of one COL IA 1 allele in an infant who died with an $\mathrm{Ol}$ phenotype that differed from the usual lethal form and had regions of increased bone density. The newborn female had dysmorphic facial features, including loss of mandibular angle. Bilateral upper and lower limb contractures were present with multiple fractures in the long bones and ribs. The long bones were not compressed and their ends were radiographically dense. She died after a few hours and histopathological studies identified extramedullary haematopoiesis in the liver, little lamellar bone formation, decreased osteoclasts, abnormally thickened bony trabeculae with retained cartilage in long bones, and diminished marrow spaces similar to those seen in dense bone diseases such as osteopetrosis and pycnodysostosis. The child was heterozygous for a COLIAl 4321G $\rightarrow$ T transversion in exon 52 that changed a conserved aspartic acid to tyrosine (D1441Y). Abnormal pro $\alpha 1$ (I) chains were slow to assemble into dimers and trimers, and abnormal molecules were retained intracellularly for an extended period. The secreted type I procollagen molecules synthesised by cultured dermal fibroblasts were overmodified along the full length but had normal thermal stability. These findings suggest that the unusual phenotype reflected both a diminished amount of secreted type I procollagen and the presence of a population of stable and overmodified molecules that might support increased mineralisation or interfere with degradation of bone.
\end{abstract}

$\mathrm{T}$ ype I collagen is the principle protein of bone, skin, ligaments, tendon, and most other connective tissues. It is synthesised as a soluble precursor, procollagen, which consists of two prool(I) chains and one proo2(I) chain. Each pro $\alpha$ chain contains a central obligatory Gly-Xaa-Yaa repeat sequence (the triple helical domain) of more than 1000 residues (in which Xaa and Yaa are any residue other than cysteine or tryptophan), and propeptides at the amino- and carboxyl-termini. The carboxyl-terminal propeptide (Cpropeptide) domains direct chain-chain recognition and alignment of pro $\alpha$ chains into correct registration. Assembled procollagen trimers are translocated from the RER to the Golgi apparatus, further modified, packaged, and secreted into the extracellular spaces where they are converted to collagen by proteolytic cleavage of the $\mathrm{N}$ - and $\mathrm{C}$-propeptides. Outside the cell, collagen trimers assemble into fibrils, which serve as the main source of mechanical strength in connective tissue and the template for matrix deposition and mineralisation in the bone.

Mutations in the COL1A1 and COL1A2 type I collagen genes perturb normal collagen assembly in the cell, secretion from the cell, and fibril assembly in the extracellular spaces, which, collectively, result in the osteogenesis imperfecta (OI) phenotypes. ${ }^{12}$ The spectrum of severity of OI ranges from lethality in the perinatal period to mild increase in the propensity for adult onset fractures. The varied clinical characteristics reflect different classes of mutations in different regions of the type I collagen genes. More than 250 different mutations (Database of Human Type I and Type III Collagen Mutations: http:// www.le.ac.uk/genetics/collagen/ $/)^{34}$ have been characterised, but how these lead to such phenotypic diversity is not well understood. The most common mutations are single base substitutions in the triple helical domain that change a codon for a glycine to a codon for another amino acid with a bulkier side chain. ${ }^{5}$ Less commonly, mutations in conserved RNA splice consensus sequences lead to partial or complete exon exclusion or intron inclusion with variations in phenotype and in the effect on both the mRNA and resultant protein. Less prevalent still have been in frame deletion or duplication mutations that involve fewer than 18 amino acids. ${ }^{6-11}$ Mutations in the C-propeptide coding region ${ }^{12-19}$ have been identified less often than other types of mutation, but show similar heterogeneity in clinical outcome. Often the latter mutations interfere with molecular assembly and slow passage of trimers through the secretory pathway and into the extracellular environment.

Proper mineral deposition is a prerequisite of strong bone and relies upon the interaction between the organic matrix, which is composed primarily of type I collagen, and calcium and phosphate in the form of hydroxyapatite. Perturbations of this interaction often lead to weak and brittle bone, such as that seen in OI when the organic matrix is abnormal ${ }^{20-23}$ or in a variety of other conditions characterised by brittle bone when the mineral component is disturbed. ${ }^{24}$ Bone is dynamic and remodelled during development and throughout life. When bone formation surpasses resorption, an increase in bone density can be the result, as seen in diseases such as

Abbreviations: Ol, osteogenesis imperfecta 

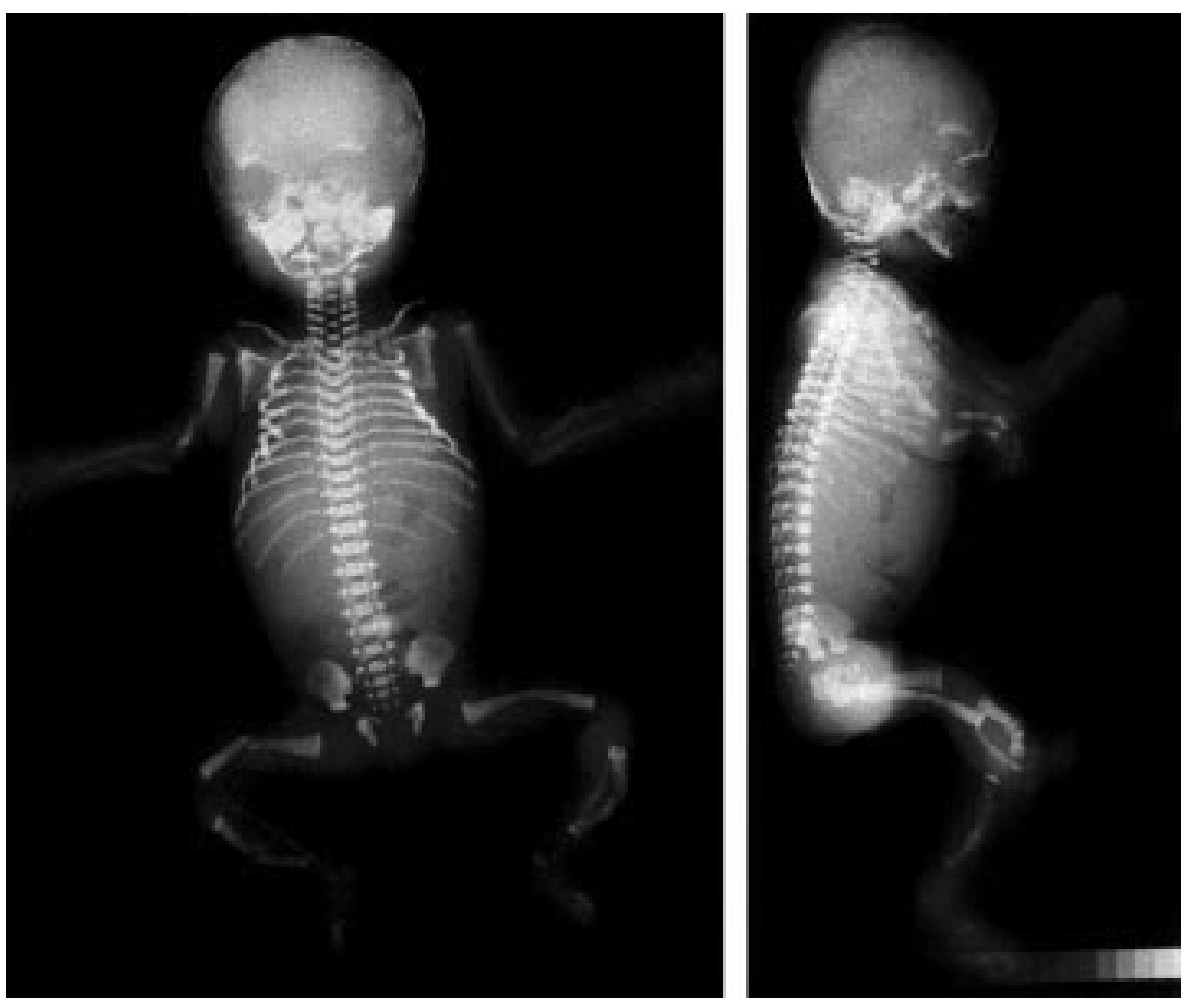

Figure $1 X$ rays of the proband. The baby sustained multiple fractures of the long bones and ribs. The cranial vault was poorly mineralised, but the base of the skull, the ends of the long bones, ribs, and spine were radiographically dense. Sternal ossification centres are prematurely visible.

osteopetrosis ${ }^{26-28}$ and pycnodysostosis. ${ }^{29}$ Osteopetrosis is characterised by hypermineralisation, narrowing of the medullary spaces, extramedullary haematopoiesis, and marked deficiency in osteoclastic activity. The known genetic causes of human osteopetrosis (which do not account for all cases of osteopetrosis) include mutations in the genes encoding carbonic anhydrase II (associated with renal tubular acidosis $)^{26}$ and the $a 3^{27}$ and TCIRG $\mathrm{l}^{28}$ subunits of the vacuolar proton pump, all of which are expressed in osteoclasts and are necessary for either dissolving inorganic bone material or degrading the organic bone matrix. Pycnodysostosis results from deficiency of the lysosomal protease, cathepsin K. Like osteopetrosis, this disorder is characterised, in part, by marked increase in bone density and diminished size of the medullary canals.

We identified a point mutation in the C-propeptide coding region of $C O L 1 A 1$ that resulted in impaired assembly of type I procollagen molecules and led to an unusual phenotype with features of both OI and dense bone disorders. Although the type I procollagen molecules made by cells from this child were overmodified along the triple helical domain, they had normal or slightly increased thermal stability which could have interfered with normal turnover of bone. This suggests that rare infants with features of dense bone diseases have mutations in the C-propeptide coding regions of type I collagen genes.

\section{METHODS}

\section{Cell culture and metabolic labelling of proteins}

Dermal fibroblast cultures were established and maintained as described previously. ${ }^{518}$ The control fibroblast cell line (A8) was from a newborn with normal type I collagen biosynthesis who died of hyaline membrane disease.

\section{Characterisation of procollagens and collagens}

Collagenous molecules were labelled overnight, pepsin digested, separated by SDS-PAGE, and analysed by two dimen-

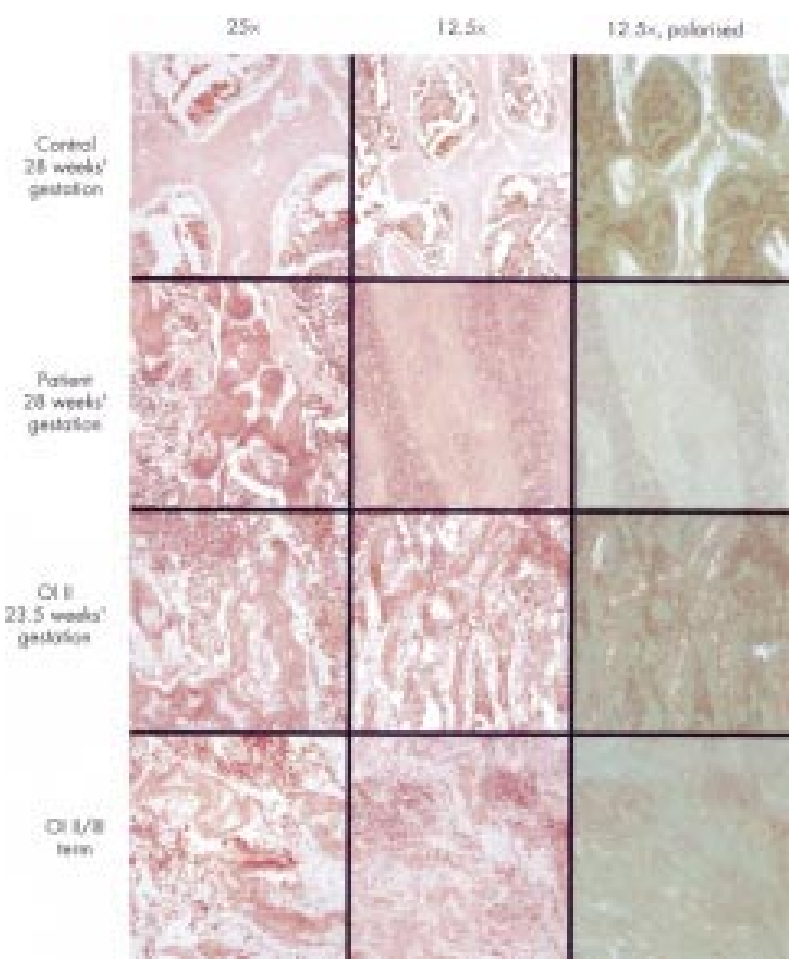

Figure 2 Histological analysis of diaphyseal bone. Decalcified bone (femur) samples from an age matched normal control, the proband, and two subjects with OI type II (gestational ages flank that of the patient) were paraffin embedded, sectioned, and stained with haematoxylin and eosin. The proband's bone differed from both normal and Ol bone, in that the bony trabeculae were poorly modelled, abnormally thick, hypercellular, and had retained cartilage cores. The patient's bone also had reduced marrow spaces and decreased osteoclasts. 
A

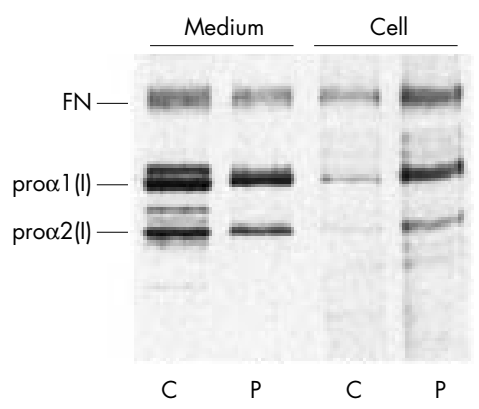

B

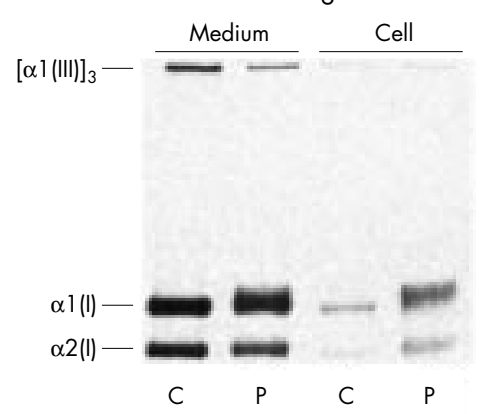

Cell

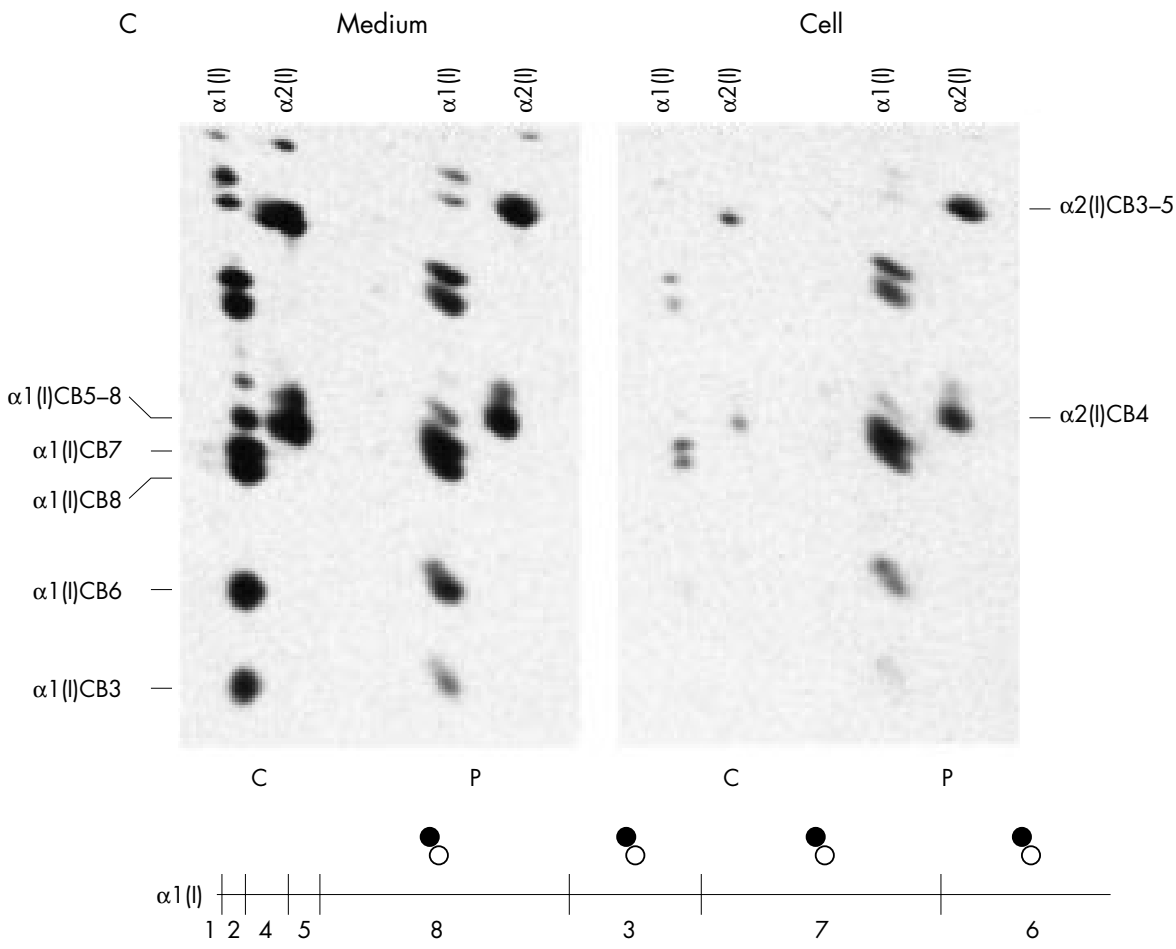

Figure 3 Analysis of procollagen and collagen. (A) Compared to control (C) cells, procollagen secretion by patient $(\mathrm{P})$ cells was reduced,

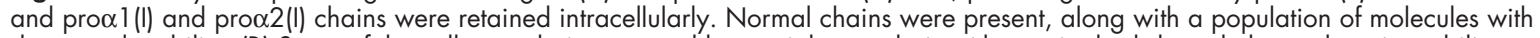
decreased mobility. (B) Some of the collagen chains, created by partial proteolysis with pepsin, had slowed electrophoretic mobility consistent with increased post-translational modification, suggesting that the helical domains were overmodified. (C) Cyanogen bromide peptides of $\alpha 1$ (I) chains were overmodified along the full extent of the molecule. The diagram below the figure represents the $\alpha 1$ (I) chain with the position of methionyl residues in the chain designated by vertical marks. The symbols above the chain designate the relative mobilities of the peptides derived from the normal chain (open circle) and the overmodified chain (filled circle).

sional mapping of cyanogen bromide fragments as previously described. ${ }^{5}$ The thermal stability of collagens was determined as described previously. ${ }^{30}$ Kinetics of molecular assembly and secretion of collagenous molecules were assayed as described previously. ${ }^{18}$

\section{Mutation detection and characterisation}

Total RNA was extracted from cultured dermal fibroblasts using the RNeasy Mini Kit ${ }^{\mathrm{TM}}$ (Qiagen). Complementary DNA was synthesised by priming with random hexamers in the presence of Superscript $\mathrm{II}^{\mathrm{TM}}$ RNase $\mathrm{H}^{-}$reverse transcriptase (GibcoBRL). Exons 42-52 from COL1A1 were sequenced using the ABI PRISM ${ }^{\mathrm{TM}}$ BigDye Terminator Cycle Sequencing Reaction Kit according to the manufacturer's recommendations (Perkin-Elmer Applied Biosystems). Parental DNA from peripheral blood was screened for the mutation by PCR amplification (sense and antisense primers were $5^{\prime}$ GGGCTTTTTGGCCAGGCCATAGTGCC-3' and 5'-TTTTGGT TTTTGGTCATGTTCGGT-3' respectively) and restriction analysis with $\mathrm{Taq}^{\alpha} \mathrm{I}$ according to the manufacturer's recommendations (New England Biolabs).

\section{RESULTS}

\section{Clinical summary}

The newborn female was born to healthy, nonconsanguineous parents who had one healthy child. There was no history of maternal infection, high blood pressure, diabetes mellitus, or exposure to teratogens. Fetal ultrasound at 28 weeks' gestation showed polyhydramnios and bowed long bones of both upper and lower limbs with healing fractures. Delivery was by caesarean section at 28 weeks' gestation 
A

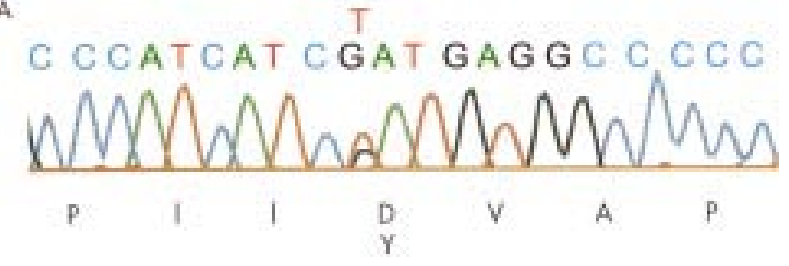

B

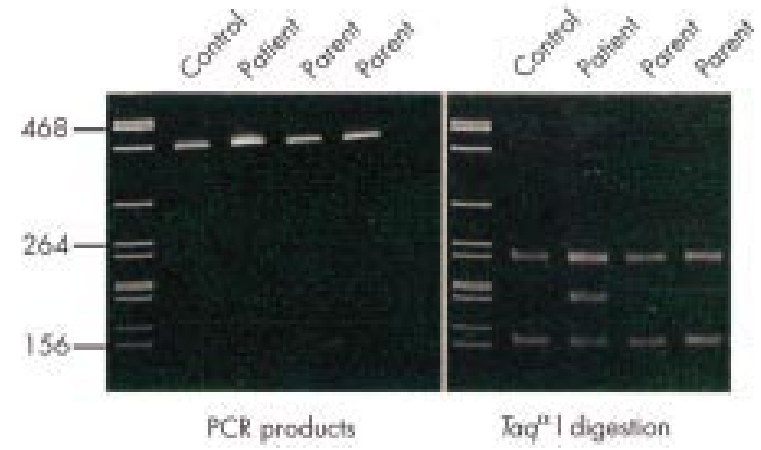

Figure 4 Mutation detection and characterisation. (A) Heterozygosity for a $4321 \mathrm{G} \rightarrow \mathrm{T}$ transversion was detected in exon 52 of COLIA 1. (B) Genomic DNA from the patient and her parents were amplified by PCR. The mutation deleted a Taq ${ }^{\alpha} \mid$ restriction site in genomic DNA and there was no evidence of the mutation in DNA from either parent indicating that the mutation occurred de novo in the affected infant. because of maternal bleeding following amniocentesis for polyhydramnios. The infant died after a few hours. She weighed $1300 \mathrm{~g}$ (90th centile) and was $36 \mathrm{~cm}$ ( < 50th centile) long. At delivery she was bradycardic and had dysmorphic facial features including loss of the mandibular angle, low set ears, soft skull, and large anterior and posterior fontanelles. $X$ rays (fig 1 ) showed multiple fractures in the long bones and ribs, and an underossified cranial vault. The skull had wormian bones and the teeth were hypoplastic. The bones of the arms and of the legs were near the normal length for gestational age and did not have the typical appearance of bones from infants with OI type II. The base of the skull, the vertebral bodies, and ends of the long bones were radiographically dense. Histologically, there was little lamellar bone formation in the skull, consistent with the radiological picture. The bony trabeculae in the shafts of the long bones were abnormally thick, hypercellular, and had retained cartilage cores, inconsistent with a typical OI type II phenotype where the trabeculae are thin (fig 2). The bone had a markedly thick periosteum, reduced marrow spaces, and decreased osteoclasts. Polarisation of the collagen in the trabeculae was decreased compared to normal polarisation in the periosteal collagen, as seen in forms of OI. The growth plate appeared normal. Additional significant findings at necropsy included hepatosplenomegaly and extramedullary haematopoiesis in the liver.

\section{Overmodification and intracellular retention of type I procollagen molecules}

Cultured dermal fibroblasts from the patient synthesised and secreted normal molecules (although less than in control strains) and a population of abnormal molecules with delayed

A

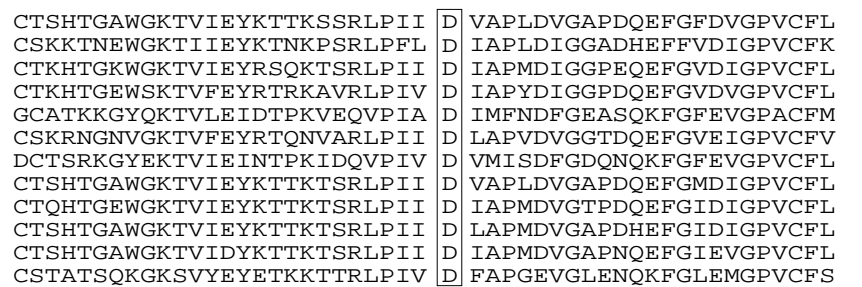

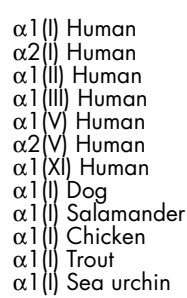

B

Prool (I) C propeptide

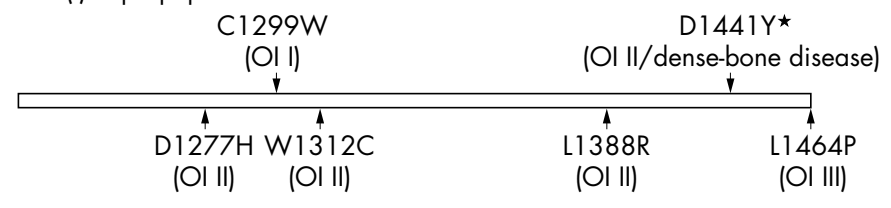

(OI II)

Figure 5 Predicted amino acid change. (A) The mutation predicts the substitution of a tyrosine residue for an aspartic acid which is conserved among all human fibrillar procollagens and several other species in which fibrillar collagens have been isolated and sequenced. (B) In the

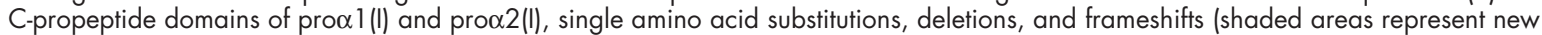
sequence) have been previously reported, which resulted in classical variants of OI (types I, II, and III). The presently described defect (*) is the only one to lead to a combined Ol and dense bone phenotype. 
A

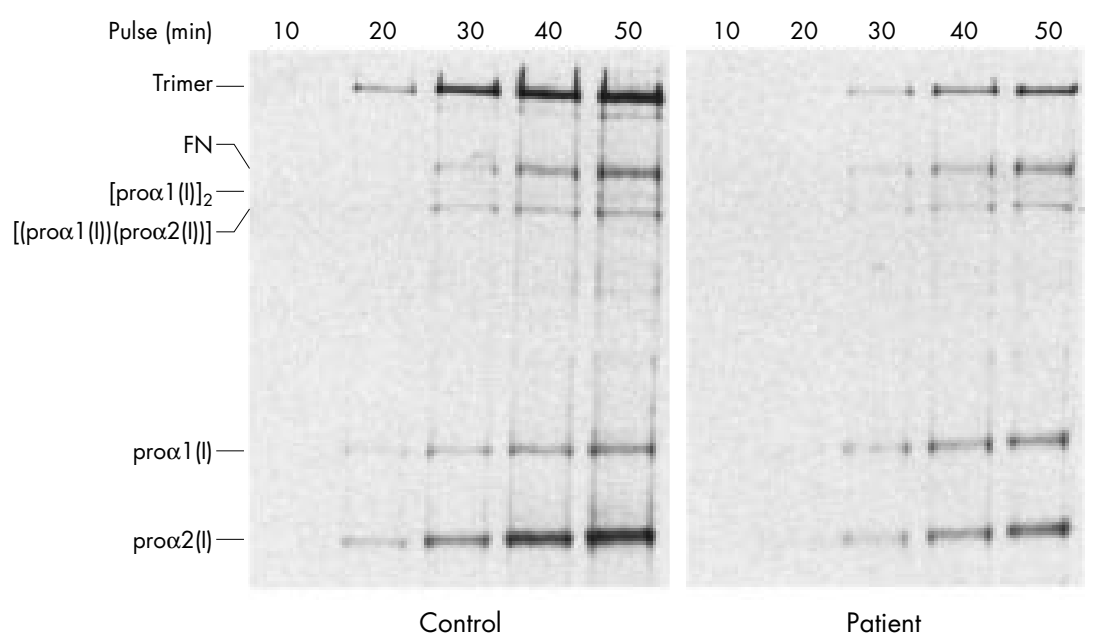

B

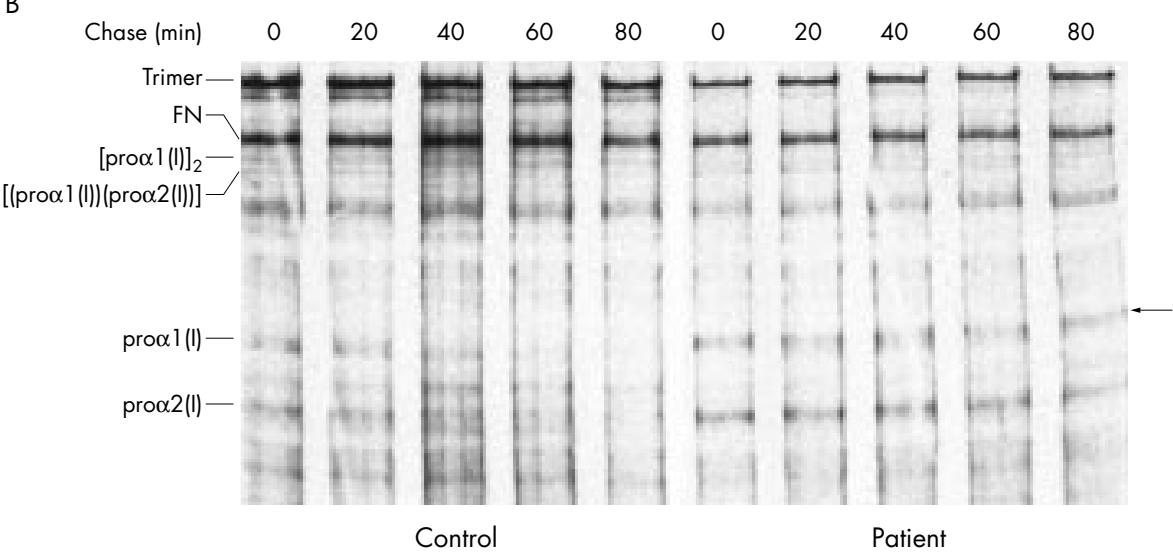

Figure 6 Kinetics of molecular assembly. (A) Cells were pulse labelled for 10, 20, 30, 40, or 50 minutes and the cell layer proteins were harvested and then separated under non-reducing conditions by SDS-polyacrylamide gel electrophoresis. In the patient's cells, assembly of dimers and trimers was delayed. (B) Monomers were chased to higher molecular weight components and out of the cell. Normal proal (I) chains from both control and patient cells assembled into dimers and trimers within 20-40 minutes. Defective pro $\alpha 1(I)$ chains $(\rightarrow)$ remained intracellularly for over 80 minutes and were slow to assemble. Excess proa2(I) was also detected.

electrophoretic mobility (fig 3A, B). The OI cells retained more $\operatorname{pro\alpha l}(\mathrm{I})$ and pro 2 (I) chains than control cells, most of which had delayed mobility. Following removal of the $\mathrm{N}$ - and C-propeptides with pepsin (fig 3B), the $\alpha \mathrm{l}$ (I) chains migrated as a broad band, consistent with increased post-translational lysyl hydroxylation and hydroxylysyl glycosylation in triple helical domains. Post-translational overmodification extended the full length of the triple helical domain (fig 3C), consistent with a mutation in the C-terminal region of the triple helix or in the C-propeptide coding regions of either chain.

\section{De novo mutation in the $\mathrm{C}$-propeptide coding region of COLIA1}

Reverse transcribed PCR fragments of the cDNA from regions that encoded the last 160 amino acids of the triple helical domain and all of the C-propeptide of COLIAI (exons 42-52) were sequenced, and heterozygosity for a transversion $(4321 \mathrm{G} \rightarrow \mathrm{T}$ ) was detected (fig $4 \mathrm{~A}$ ). The mutation changed the aspartic acid at position 1441 of the prool (I) chain to tyrosine (D1441Y) 24 residues from the carboxyl-terminus of the chain. The mutation deleted a Taq ${ }^{\alpha}$ I restriction site. There was no evidence of the mutation in parental DNA, indicating that the mutation occurred de novo (fig 4B). The C-propeptide domain is similar in all fibrillar collagens and D1441 is one of a few residues absolutely conserved in all C-propeptide sequences analysed (fig 5A). Previously reported defects in the
C-propeptide domain of prool(I) and proo2(I) led to mild (type I) ${ }^{17-19}$, lethal (type II), ${ }^{12-14}$ and progressively deforming (type III $)^{15}{ }^{16}$ OI, but only the presently described abnormality resulted in a combined phenotype of OI with regions of dense bone (fig 5B).

\section{Slowed assembly and secretions of type I procollagen}

Since the C-propeptide directs procollagen assembly, we examined the rate at which prool(I) chains assembled into dimers and trimers. In control cells, by 20 minutes, labelled prool(I) and $\operatorname{pro} \alpha 2(\mathrm{I})$ chains were assembled into dimers (pro $\alpha \mathrm{l}(\mathrm{I})_{2}$ and pro $\alpha 1(\mathrm{I})$-pro $\alpha 2(\mathrm{I})$ ) and trimers; the amount of trimer peaked at 40 minutes (fig 6A). In the patient's cells, collagenous proteins were slowly synthesised, and assembly of dimer and trimer species was delayed with levels detectable only after 30 minutes of labelling. To determine how long the abnormal proo chains remained unincorporated into complexes, proteins were labelled for 80 minutes and were then chased for up to 80 minutes with excess non-labelled proline (fig 6B). Normal proal(I) chain monomers in both control and patient cells disappeared within 20-40 minutes. In contrast, defective chains with delayed electrophoretic mobility persisted as monomers for over 80 minutes and were not rapidly degraded.

\section{Thermal stability of defective type I collagen}

Although abnormal pro $\alpha$ chains were slow to assemble into molecules and became post-translationally overmodified, 

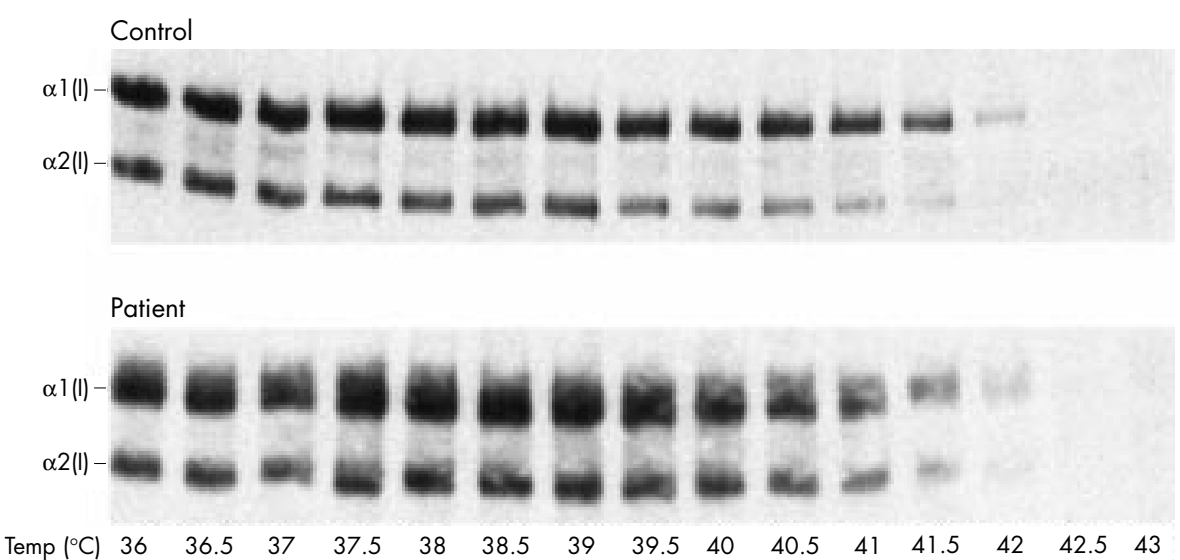

Figure 7 Thermal stability of abnormal collagens. Secreted collagens from Ol cells had the same melting temperature as normal collagens. Thus, slowed assembly and increased modification did not affect helix stability.

some formed pepsin resistant trimers that were secreted. The secreted overmodified molecules had a melting temperature $\left(\mathrm{T}_{\mathrm{m}}\right)$ similar to those of the normal molecules made by the same cells and of the normal molecules made by control cells (fig 7). Thus, although the C-propeptide defect reduced both the rate and efficiency of trimer formation and led to increased hydroxylation and glycosylation of the pro $\alpha$ chains, neither the mutation nor overmodification altered the structure of the triple helix as judged by helix stability.

\section{DISCUSSION}

Osteogenesis imperfecta is a heterogeneous disorder with clinical characteristics that include bone fragility and deformity, alterations in scleral hue, dentinogenesis imperfecta, hearing loss, soft tissue dysplasia, diminished stature, and decreased bone mineralisation. ${ }^{31-33}$ These clinical features, along with radiological and genetic criteria, were used to separate four major clinical types of OI (types I, II, III, and IV).$^{31}$ Although the infant described here died in the perinatal period following delivery at 28 weeks' gestation, the radiographic features are distinct from those that characterise the perinatal lethal form of OI, OI type II..$^{25} 3134$ She did have markedly diminished calvarial mineralisation with Wormian bones as seen in OI type II. However, her long bones were straight, non-compressed, and about the length expected for gestational age, the ribs had near normal shape, but were beaded, and the vertebral body morphology was normal rather than flat. While the radiographic features resembled those of OI type III, the near normal chest size and the straight bones distinguish the presentation. Radiographically, the infant had dense ribs, vertebral bodies, and increased bone density at the base of the skull and ends of the long bones. She also had narrow medullary cavities, hepatosplenomegaly owing to extramedullary haematopoiesis, and decreased numbers of osteoclasts, all of which can be features of osteopetrosis and pycnodysostosis, and further distinguish this infant from the usual forms of OI.

Only a few mutations in the C-propeptide coding regions of type I collagen genes have been identified..$^{12-19}$ Some of these mutations result in premature termination codons and the mRNA is unstable or, if stable, the peptide chain is either unstable and rapidly degraded or fails to associate in trimers. ${ }^{12}{ }^{17}{ }^{19}$ In these cases, the phenotype reflects the size of the pool from which normal molecules can be assembled. Other C-propeptide mutations, ${ }^{13-15} 18$ including the one described here, effect procollagen assembly, structure, and secretion. In general, trimer assembly is delayed, secretion diminished, and the total amount of procollagen produced may be reduced. The aspartic acid at position 1441 is conserved among all fibrillar propeptides and lies outside the putative chain selection region, ${ }^{35}$ suggesting that the deleterious effect of the presently described defect is on intrachain folding rather than chain selectivity. Delayed assembly and increased modifications did not prevent synthesis of thermally stable molecules, indicating a normal relationship between neighbouring chains in the trimer, in contrast to the effects of mutations in the triple helical domain. ${ }^{36}$ In all these instances, it is not clear what alterations occur in the triple helical molecule, once procollagen is completely folded.

Our findings suggest that the combined phenotype of OI and dense bone disease in this infant reflect both a diminished amount of secreted type I procollagen and the presence of a population of stable and overmodified molecules that might support increased mineralisation or interfere with degradation of bone during remodelling. However, this alone is not adequate to explain the observed phenotype in light of the fact that other C-propeptide defects lead to similar biochemical findings, but very different phenotypes. In these other cases, cells synthesised and secreted stable and overmodified molecules, just as we observed here. Yet these mutations led to mild OI type $\mathrm{I}^{17}{ }^{18}$ lethal OI type $\mathrm{II},{ }^{13}$ and severe OI type $\mathrm{III},{ }^{15}$ and none of these cases had increased bone thickness and density. This suggests the existence of novel mechanisms, other than excess modification and diminished levels of normal procollagen, contributing to the pathogenesis of OI in these cases, leading to the varied phenotypes.

We hypothesise that the C-propeptide of type I procollagen, in addition to its function as mediator of collagen assembly in the secretory pathway, acts as a signalling molecule in the extracellular matrix, and that structural defects hamper this activity and contribute to the pathogenesis of OI. In support of this hypothesis, studies have shown that C-propeptide suppresses collagen synthesis in fibroblasts, ${ }^{37}$ initiates growth arrest and differentiation of cultured Schwann cells, ${ }^{38}$ and suppresses collagen synthesis of osteoblastic cells at the early differentiated stage. ${ }^{39} 40$ Thus, the unique phenotype of the patient described here and the variance in phenotypic traits between patients with other C-propeptide mutations may reflect structural differences of the C-propeptides and their altered abilities to communicate with cells in the extracellular matrix. It might therefore be prudent to consider the effects that defects in the C-propeptide may have on signalling roles of this domain when describing the molecular pathogenesis of some types of OI.

\section{ACKNOWLEDGEMENTS}

We thank Dr Deborah Krakow, Cedars-Sinai Medical Center, Los Angeles, CA for analysing the radiographs and critically reviewing the 
manuscript. This work was supported, in part, by a grant from the USPHS (AR 21557). James Pace was supported by the Molecular Training Program in Cancer Research (NIH T32 CA 09437) at the time this research was conducted. William Wilcox is supported by grants 5P01-HD22657 and M01-RR00425.

\section{Authors' affiliations}

J M Pace, M Atkinson, U Schwarze, P H Byers, Department of Pathology, University of Washington, Seattle, WA, USA D Chitayat, Department of Obstetrics and Gynecology, Mount Sinai Hospital and Department of Pediatrics, The Hospital for Sick Children, Department of Laboratory Medicine and Pathobiology, University of Toronto, Toronto, Ontario, Canada

W R Wilcox, Division of Medical Genetics, Ahmanson Department of Pediatrics, Steven Spielberg Pediatric Research Center, Burns and Allen Research Institute, Cedars-Sinai Medical Center, Department of Pediatrics, UCLA School of Medicine, Los Angeles, CA, USA P H Byers, Department of Medicine, University of Washington, Seattle, WA, USA

\section{REFERENCES}

1 Byers PH. Disorders of collagen biosynthesis and structure. In: Scriver CR, Beaudet AC, Sly WS, Valle D, Childs B, Kinzler KW, Vogelstein B, eds. The metabolic and molecular bases of inherited disease. New York: McGraw-Hill, 2001:5241-85.

2 Myllyhariu J, Kivirikko KI. Collagens and collagen-related diseases. Ann Med 2001; 33:7-21

3 Dalgleish R. The human type I collagen mutation database. Nucleic Acids Res 1997:25:181-7.

4 Dalgleish R. The Human Collagen Mutation Database. Nucleic Acids Res 1998;26:253-5.

5 Bonadio J, Byers PH. Subtle structural alterations in the chains of type I procollagen produce osteogenesis imperfecta type II. Nature 1985:316:363-6

6 Hawkins JR, Superti-Furga A, Steinmann B, Dalgleish R. A 9-base pair deletion in COLIAl in a lethal variant of osteogenesis imperfecta. J Biol Chem 1991;266:22370-4.

7 Lund AM, Schwartz M, Skovby F. Variable clinical expression in a family with Ol type IV due to deletion of three base pairs in COLIA I. Clin Genet 1996;50:304-9.

8 Molyneux K, Starman BJ, Byers PH, Dalgleish R. A single amino acid deletion in the alpha $2(I)$ chain of type I collagen produces osteogenesis imperfecta type III. Hum Genet 1993;90:62 1-8.

9 Horwitz EM, Prockop DJ, Fitzpatrick LA, Koo WW, Gordon PL, Neel M, Sussman M, Orchard P, Marx JC, Pyeritz RE, Brenner MK. Transplantability and therapeutic effects of bone marrow-derived mesenchymal cells in children with osteogenesis imperfecta. Nat Med 1999:5:309-13.

10 Wallis GA, Kadler KE, Starman BJ, Byers PH. A tripeptide deletion in the triple-helical domain of the pro 1 I (I) chain of type I procollagen in a patient with lethal osteogenesis imperfecta does not alter cleavage of the molecule by N-proteinase. J Biol Chem 1992;267:25529-34

11 Pace JM, Atkinson M, Willing MC, Wallis G, Byers PH. Deletions and duplications of Gly-Xaa-Yaa triplet repeats in the triple helical domains of type I collagen chains disrupt helix formation and result in several types of osteogenesis imperfecta. Hum Mutat 2001;18:319-26.

12 Bateman JF, Lamande SR, Dahl HH, Chan D, Mascara T, Cole WG. A frameshift mutation results in a truncated nonfunctional carboxyl-terminal proa 1 (I) propeptide of type I collagen in osteogenesis imperfecta. J Biol Chem 1989;264:10960-4.

13 Chessler SD, Wallis GA, Byers PH. Mutations in the carboxyl-terminal propeptide of the proa I (I) chain of type I collagen result in defective chain association and produce lethal osteogenesis imperfecta. J Biol Chem 1993:268:18218-25.

14 Chessler SD, Byers PH. BiP binds type I procollagen pro $\alpha$ chains with mutations in the carboxyl-terminal propeptide synthesized by cells from patients with osteogenesis imperfecta. J Biol Chem 1993;268: 18226-33.

15 Oliver JE, Thompson EM, Pope FM, Nicholls AC. Mutation in the carboxy-terminal propeptide of the pro 1 I II) chain of type I collagen in a child with severe osteogenesis imperfecta (Ol type III): possible implications for protein folding. Hum Mutat 1996;7:318-26.

16 Pihlajaniemi T, Dickson LA, Pope FM, Korhonen VR, Nicholls A, Prockop DJ, Myers JC. Osteogenesis imperfecta: cloning of a pro- $\alpha 2(I)$ collagen gene with a frameshift mutation. J Biol Chem 1984;259:12941-4.

17 Willing MC, Cohn DH, Byers PH. Frameshift mutation near the $3^{\prime}$ end of the COLIAl gene of type I collagen predicts an elongated proo 1 (I) chain and results in osteogenesis imperfecta type I (published erratum appears in J Clin Invest 1990;85 following 1338). J Clin Invest $1990 ; 85: 282-90$
18 Pace JM, Kuslich CD, Willing MC, Byers PH. Disruption of one intra-chain disulphide bond in the carboxyl-terminal propeptide of the prool (I) chain of type I procollagen permits slow assembly and secretion of overmodified, but stable procollagen trimers and results in mild osteogenesis imperfecta. J Med Genet 2001:38:443-9.

19 Willing MC, Deschenes SP, Slayton RL, Roberts EJ. Premature chain termination is a unifying mechanism for COLIAl null alleles in osteogenesis imperfecta type I cell strains. Am J Hum Genet 1996;59:799-809.

20 Cohen-Solal L, Zylberberg L, Sangalli A, Gomez Lira M, Mottes M. Substitution of an aspartic acid for glycine 700 in the alpha 2(I) chain of type I collagen in a recurrent lethal type II osteogenesis imperfecta dramatically affects the mineralization of bone. J Biol Chem 1994;269:14751-8

21 Culbert AA, Lowe MP, Atkinson M, Byers PH, Wallis GA, Kadler KE. Substitutions of aspartic acid for glycine-220 and of arginine for glycine-664 in the triple helix of the $\operatorname{pro\alpha } 1$ (I) chain of type I procollagen produce lethal osteogenesis imperfecta and disrupt the ability of collagen fibrils to incorporate crystalline hydroxyapatite. Biochem J 1995;311 (Pt 3):815-20

22 Jones SJ, Glorieux FH, Travers R, Boyde A. The microscopic structure of bone in normal children and patients with osteogenesis imperfecta: a survey using backscattered electron imaging. Calcif Tissue Int $1999 ; 64: 8-17$

23 Traub W, Arad T, Vetter U, Weiner S. Ultrastructural studies of bones from patients with osteogenesis imperfecta. Matrix Biol 1994;14:337-45.

24 Streeten EA, Marx SJ, Levine MA, Vitamin D metabolism or action. In: Emery AEH, Rimoin DL, Connor JM, Pyeritz RE, eds. Emery and Rimoin's principles and practice of medical genetics. New York: Churchill Livingstone, 1997:1991-2007.

25 Sillence DO. Disorders of bone density, volume, and mineralization. In: Emery AEH, Rimoin DL, Connor JM, Pyeritz RE, eds. Emery and Rimoin's principles and practice of medical genetics. New York: Churchill Livingstone, 1997:2817-35

26 Roth DE, Venta PJ, Tashian RE, Sly WS. Molecular basis of human carbonic anhydrase II deficiency. Proc Natl Acad Sci USA 1992:89:1804-8.

27 Kornak U, Schulz A, Friedrich W, Uhlhaas S, Kremens B, Voit T, Hasan $C$, Bode U, Jentsch TJ, Kubisch C. Mutations in the a3 subunit of the vacuolar $\mathrm{H}(+)-$ ATPase cause infantile malignant osteopetrosis. Hum $\mathrm{Mol}$ Genet 2000;9:2059-63

28 Frattini A, Orchard PJ, Sobacchi C, Giliani S, Abinun M, Mattsson JP, Keeling DJ, Andersson AK, Wallbrandt P, Zecca L, Notarangelo LD, Vezzoni P, Villa A. Defects in TCIRG1 subunit of the vacuolar proton pump are responsible for a subset of human autosomal recessive osteopetrosis. Nat Genet 2000;25:343-6.

29 Gelb BD, Bromme D, Desnick RJ. Pycnodysostosis: cathepsin K deficiency. In: Scriver CR, Beaudet AC, Sly WS, Valle D, Childs B, Kinzler KW, Vogelstein B, eds. The metabolic and molecular bases of inherited disease. New York: McGraw-Hill, 2001:3453-68.

30 Bulleid NJ, Wilson R, Lees JF. Type-III procollagen assembly in semi-intact cells: chain association, nucleation and triple-helix folding do not require formation of inter-chain disulphide bonds but triple-helix nucleation does require hydroxylation. Biochem J 1996;317/ $\mathrm{Pt}$ 1):195-202.

31 Sillence DO, Senn A, Danks DM. Genetic heterogeneity in osteogenesis imperfecta. J Med Genet 1979;16:101-16.

32 Sillence DO, Rimoin DL, Danks DM. Clinical variability in osteogenesis imperfecta -variable expressivity or genetic heterogeneity. Birth Defects 1979:15:113-29.

33 Tsipouras P. Osteogenesis imperfecta. In: Beighton P, ed. McKusick's heritable disorders of connective tissue. St Lovis: Mosby, 1993:281-314

34 Byers PH, Tsipouras P, Bonadio JF, Starman BJ, Schwartz RC. Perinatal lethal osteogenesis imperfecta (OI type II): a biochemically heterogeneous disorder usually due to new mutations in the genes for type I collagen. Am J Hum Genet 1988;42:237.

35 Lees JF, Tasab M, Bulleid NJ. Identification of the molecular recognition sequence which determines the type-specific assembly of procollagen. EMBO J 1997: 16:908-16

36 Bonadio J, Holbrook KA, Gelinas RE, Jacob J, Byers PH. Altered triple helical structure of type I procollagen in lethal perinatal osteogenesis imperfecta. J Biol Chem 1985;260: 1734-42.

$37 \mathrm{Wu} \mathrm{CH}$, Donovan $\mathrm{CB}, \mathrm{Wu} \mathrm{GY}$. Evidence for pretranslational regulation of collagen synthesis by procollagen propeptides. J Biol Chem 1986;261:10482-4

38 Rushton JA, Schmitz S, Gunn-Moore F, Sherman D, Pappas CA, Ritchie $J M$, Haynes LW. Growth arrest and spontaneous differentiation are initiated through an autocrine loop in clonally derived Schwann cells by al-procollagen I C-propeptide. J Neurochem 1999:73:1816-27.

39 Mizuno M, Fujisawa R, Kuboki Y. Carboxyl-terminal propeptide of type I collagen (c-propeptide) modulates the action of TGF-beta on MC3T3-E 1 osteoblastic cells. FEBS Lett 2000;479:123-6.

40 Mizuno M, Fujisawa R, Kuboki Y. The effect of carboxyl-terminal propeptide of type I collagen (c-propeptide) on collagen synthesis of preosteoblasts and osteoblasts. Calcif Tissue Int 2000;67:391-9. 